Mots. Les langages du politique

Discours d'autorité : des discours sans éclat(s)?

\title{
Construire et déconstruire l'autorité en discours. Le figement discursif et sa subversion
}

Construction and deconstruction of the authority phenomena in discourse. The discursive collocate and its subversion

Construir y desconstruir la autoridad en el discurso. La fijación discursiva y su subversión

\section{Alice Krieg-Planque}

\section{OpenEdition}

Journals

Édition électronique

URL : https://journals.openedition.org/mots/21926

DOI : $10.4000 /$ mots. 21926

ISSN : 1960-6001

Éditeur

ENS Éditions

\section{Édition imprimée}

Date de publication : 23 mars 2015

Pagination : 115-132

ISBN : 978-2-84788-698-6

ISSN : 0243-6450

\section{Référence électronique}

Alice Krieg-Planque, « Construire et déconstruire l'autorité en discours. Le figement discursif et sa subversion », Mots. Les langages du politique [En ligne], 107 | 2015, mis en ligne le 23 mars 2017 consulté le 22 avril 2022. URL : http://journals.openedition.org/mots/21926 ; DOI : https://doi.org/ $10.4000 /$ mots. 21926 


\section{Alice Krieg-Planque}

\section{Construire et déconstruire l'autorité en discours. Le figement discursif et sa subversion}

Dans cet article, nous nous intéressons aux discours d'autorité dans leur rapport à la question du figement. En effet, il nous apparaît que le figement, entendu au sens large et général que nous lui donnerons ici, participe à la capacité des discours à faire autorité, c'est-à-dire à dissuader la contradiction, à s’imposer sur le mode de l'évidence, à sembler faire consensus.

En écho à l'interrogation qui est mise en discussion dans l'intitulé de ce dossier de la revue Mots. Les langages du politique, nous répondons par l'affirmative : les discours d'autorité se présentent bien comme des «discours sans éclats", au sens où, de la façon la plus littéralement formelle, ils ne présentent ni aspérité ni saillie. Les discours d'autorité sont des discours dont la linéarité a été travaillée de manière que ni l'imprévu ni les débordements n'y trouvent plus place : les discours d'autorité sont constitués, à différents égards, d'énoncés stabilisés.

Selon un certain sentiment rhétorique assez partagé, l'absence de relief qui caractérise les discours d'autorité fait de ce type de production verbale des "discours sans éclat», au sens où l'absence d'aspérité confère aux discours d'autorité un aspect terne, propre à susciter l'ennui (les locuteurs parleront alors volontiers de «discours soporifiques»). Selon un autre sentiment rhétorique ordinaire, de substrat plus critique que le précédent, et qui en reprend les conclusions mais qui les dépasse, l'absence d'aspérité formelle présentée par les discours d'autorité est l'étayage, dans la langue, de leur dimension doxique au plan de l'argumentation (les locuteurs évoqueront alors des « discours anesthésiants ", au sens où ils endorment l'esprit critique). Nous retiendrons les observations empiriques de ce dernier sentiment, dont nous tenterons d'expliciter le soubassement linguistiquement fondé, au moyen d'illustrations et d'exemples qui étayent notre propos.

La notion de figement sera le fil conducteur de notre réflexion dans cette étude, laquelle se présente en trois temps. Dans une première partie, nous soulignons combien la notion de figement est pertinente pour appréhender les

Université Paris-Est Créteil (UPEC), Ceditec (EA 3119)

krieg-planque@u-pec.fr

Mots. Les langages du politique $\mathrm{n}^{\circ} 107$ mars $2015 \bullet 115$ 
phénomènes d'autorité en discours. Nous nous appuyons pour cela à la fois sur les observations de témoins particulièrement éclairés et sur les travaux qui ont fondé l'analyse du discours politique en France. Dans une seconde partie, nous montrons comment, à travers différents instruments et dispositifs qu'elles mettent en place, les organisations entreprennent de limiter l'espace du dicible et de contrôler l'étendue de la créativité verbale. Nous convoquons pour cette démonstration deux séries d'exemples, qui renvoient successivement à la terminologie et à l'autorégulation. Dans une dernière partie, nous nous intéressons à des locuteurs qui, comme en réponse aux précédents, adoptent explicitement une démarche de critique politique ou idéologique. Nous observons que, pour ces locuteurs qui adoptent un point de vue critique, la mise en cause de l'autorité des discours adverses passe par une subversion de leur caractère figé. Là encore, nous nous appuyons sur des illustrations précises, qui correspondent à deux types de genres ou dispositifs mis en œuvre par ces locuteurs : les générateurs d'énoncés et les représentations visuelles (cartes, plans...) du vocabulaire supposé dominant.

\title{
Le cadre de l'analyse : le figement et l'autorité du discours
}

\author{
De précieux observateurs de l'autorité en train de se faire \\ ou de se défaire : deux témoignages
}

Différents observateurs particulièrement avisés ont repéré une relation entre discours d'autorité et prévisibilité des énoncés. Nous évoquerons ici Hannah Arendt et Victor Klemperer, dans les écrits desquels se trouve signalé un tel lien. Ces deux auteurs sont envisagés, dans le cas présent, comme des témoins dont le sens de l'observation est singulièrement aiguisé, mais qui expriment un sentiment rhétorique spontané sans doute assez partagé.

Alors qu'elle couvre le procès d'Adolf Eichmann à Jérusalem, en 1961, Hannah Arendt porte attention à la personnalité d'Eichmann, à ses faits et gestes, à ses attitudes, à ses façons de parler. Sur ce dernier point, Arendt relève à plusieurs reprises le caractère stéréotypé des propos du criminel nazi. Commentant une réaction d'Eichmann à l'un de ses juges, Arendt écrit (1963, p. 84-86):

Eichmann s'excusa en disant : "Le langage administratif (Amtssprache) est le seul que je connaisse. » Mais ce qu'il faut remarquer ici, c'est que le langage administratif était devenu le seul qu'il connût parce qu'il était réellement incapable de prononcer une seule phrase qui ne fût pas un cliché. [...] Qu'il écrivît ses mémoires en Argentine ou à Jérusalem, qu'il s'adressât au policier qui l'interrogeait ou au tribunal, il disait toujours la même chose, avec les mêmes mots. Plus on l'écoutait, plus on se rendait à l'évidence que son incapacité à s'exprimer était étroitement liée à son incapacité à penser. 
Chez Eichmann, la soumission à l'autorité, comme renoncement à penser par soi-même, s'accompagne ainsi d'une dépersonnalisation de l'expression, qui se trouve réduite à des clichés langagiers. Celui qu'Arendt identifie par ailleurs comme étant avant tout un bureaucrate zélé et un fonctionnaire du totalitarisme est donc aussi un homme qui a abandonné sa faculté de créativité verbale au pouvoir en place.

Dans la partie de son journal rédigée de 1933 à 1945, Victor Klemperer témoigne de ce qu'il observe depuis la prise du pouvoir par les nazis jusqu'à l'effondrement du Troisième Reich. En avril 1945, ayant fui sous les bombardements la ville de Dresde où il avait habité durant toute la guerre, Klemperer se trouve à errer par villes et campagnes, jusqu'en Bavière. Il note en détail les formes prises par le Troisième Reich en pleine dislocation : horloges que plus personne ne règle et qui marquent des heures différentes, civils inquiets qui colportent des rumeurs sur la possible mort du Führer, soldats déconfits qui hésitent à quitter l'uniforme et cherchent désespérément à savoir qui incarne désormais l'autorité, combattants en attente de directives... À la date du 9 avril, soit une vingtaine de jours avant le suicide d'Adolf Hitler, Victor Klemperer et son épouse Eva croisent, à Munich, un jeune couple désemparé dont Klemperer parle ainsi (1995, p. 685) :

Ils venaient de Graz, l'homme, petit fonctionnaire, était en route pour Berlin dans le but de réclamer ses arriérés de salaire; ils ne savaient pas où ils allaient pouvoir trouver le calme (momentané) ni où ils allaient être «affectés». Il était amusant de voir comment, dans la tête désillusionnée et amère de l'homme, quelques bribes de $L T /$ sagement apprises, comme il se doit, surnageaient encore mollement çà et là comme des îlots en train de se désagréger.

Quelques lignes plus loin, Klemperer rapporte, au discours indirect libre, des propos du jeune couple qui se plaint et s'inquiète (ibid., p. 686) :

Et c'était encore une fois aux petits de trinquer, de payer les pots cassés, tout : la misère, la guerre... Mais, bien entendu, tout finira par s'arranger. Puisque le Führer a dit que rien n'est impossible, et puis : nous sommes effectivement « le rempart de l’Europe contre le bolchévisme».

Les guillemets posés par Klemperer signalent les bribes de LTI qui surnagent encore dans le discours des jeunes sujets du Reich dans le désarroi. Dans son journal, Klemperer avait déjà relevé que «être affecté » était une locution caractéristique de la langue du Troisième Reich (ironiquement appelée la LTI, ou Lingua Tertii Imperii). La qualification du régime hitlérien de «rempart de l’Europe contre le bolchévisme » est également une expression récurrente dans les discours nazis. Ce jeune homme et cette jeune femme sur lesquels le pouvoir nazi avait prise assuraient durant la guerre la transmission des régularités de discours caractéristiques du régime. En 1945, l'effondrement de ce régime amène à une désagrégation de ces régularités, réintroduisant peu à peu pour les sujets parlants la possibilité de créations langagières inédites. 
Comme en miroir d'Hannah Arendt qui observe que la reconnaissance de l'autorité s'accompagne d'une reproduction du discours qui incarne cette autorité, Victor Klemperer remarque que le reflux de l'autorité s'accompagne d'un relâchement de la contrainte de prévisibilité des énoncés.

\section{La notion de figement : proposition de définition}

Ces observations nous encouragent à mobiliser une notion qui rende compte des intuitions consignées par Arendt et Klemperer, tout en nous permettant d'aller au-delà d'expressions justes mais un peu vagues telles que «prévisibilité des énoncés» ou «régularités de discours».

Cette notion est le figement, que nous proposons d'appréhender dans la continuité des travaux sur le sujet, tout en retenant une conception suffisamment large pour qu'elle puisse constituer un principe général guidant l'analyse en différentes circonstances. Le figement comme phénomène observable, en effet, s'appuie sur des propriétés très abstraites de la langue comme système. Par conséquent, son analyse est susceptible d'être menée pour des études les plus diverses. Selon la visée généralisante qui nous anime ainsi, nous définissons le figement comme une occasion de resserrement de la créativité verbale, le principe d'imprévisibilité inhérent à l'activité de langage (récursivité, commutabilité, ouverture de la combinatoire...) se trouvant alors restreint. Une telle conception du figement permet d'aborder des unités de divers ordres, sur lesquelles des occasions de resserrement peuvent à chaque fois être observées : unités lexicales (noms composés, unités polylexicales...), unités locutionnelles (expressions idiomatiques, routines d'écriture...), unités phrastiques (proverbes, maximes...), voire unités textuelles (genres de discours, textes patrimoniaux...).

Dans toute sa généralité, la notion de figement ainsi définie permet de rendre compte de la manière dont l'autorité en discours renvoie à des modes d'expression spécifiques, dont nous verrons dans les parties suivantes que les locuteurs s'emploient tantôt à les produire, tantôt à les déconstruire. Ces opérations de production et de déconstruction seront aussi pour nous l'occasion de souligner que le figement et le défigement ne sont pas uniquement des faits de langue, linguistiquement descriptibles, mais aussi des pratiques sociales que des acteurs prennent en charge, dans des logiques organisationnelles ou politiques.

\section{Le figement comme objet inaugural de l'analyse du discours politique : la question de l'idéologie}

À travers une terminologie diversifiée (cooccurrents immédiats, segments répétés, phraséologie, sloganisation...), la question du resserrement de la créati- 
vité verbale a été repérée comme un thème essentiel dès les commencements de l'analyse du discours politique en France, dans ce que l'on a pu appeler l' «École française d'analyse du discours » : le figement, qu'il soit étudié ou non sous ce nom, apparaît comme un phénomène qui retient dès le départ l'attention des analystes dès lors que ceux-ci étudient les discours politiques, syndicaux et d'appareils.

Dans le cadre du laboratoire de Lexicométrie et textes politiques de SaintCloud, Pierre Lafon et André Salem développent la pratique dite des «segments répétés» (Salem, 1987). Reposant sur une approche strictement probabiliste du discours, et appréhendant celui-ci comme une succession de formes, l'étude des segments répétés permet de faire surgir des régularités caractéristiques du locuteur dans un contexte de prise de parole déterminé. Par exemple, le segment répété « la lutte de classe et de masse » apparaît comme l'une des formulations syntagmatiques répétitives des discours de certaines centrales syndicales au milieu des années soixante-dix. Par la mise en évidence, grâce à la statistique textuelle, de segments répétés, il est ainsi possible d'identifier les formulations récurrentes typiques d'un locuteur, d'une période, d'un registre, d'un genre de discours, etc.

À la même époque, Pierre Fiala s'intéresse à la phraséologie, dont il lui semble qu'elle gagne à être étudiée, de manière souple, sous l'angle des unités phraséologiques. Par « unités phraséologiques», il entend désigner «des figements, c'est-à-dire des ensembles plus ou moins longs de formes simples construites dans des contextes contraints, susceptibles néanmoins de certaines variations » (Fiala, 1987, p. 32). En s'intéressant à la phraséologie et aux unités phraséologiques, Pierre Fiala suggère de montrer comment, au-delà des stricts segments répétés, les discours politiques sont traversés par des formes variées de répétition et marqués par des solidarités discontinues mais récurrentes entre unités lexicales.

Plaidant également pour une conception large de la régularité des réalisations langagières, Maurice Tournier (1985) propose la notion de «sloganisation », par laquelle il désigne le degré de figement et de répétitivité que présente un texte, et par lequel sont tissées les redondances organisées dont le discours politique est coutumier.

Ces différentes catégories d'analyse sont indissociables des équipements techniques qui permettent de les faire apparaître : l'intérêt pour les phénomènes de figement tel que décrit ci-dessus va en effet de pair avec les premiers développements de l'analyse statistique des données textuelles, pratiquée d'abord à l'aide de cartes perforées, puis entièrement informatisée. La statistique textuelle permet ainsi d'objectiver ce qui, sous les regards attentifs d'Hannah Arendt et de Victor Klemperer, relevait avant tout de l'intuition.

Du point de vue des chercheurs des années soixante / quatre-vingt que nous venons d'évoquer, les phénomènes observés ne sont en rien les témoignages 
d'un aspect anodin du langage : les phénomènes de figement que l'analyse automatisée des données textuelles permet de rendre visibles sont considérés comme des « révélateurs» de la position idéologique des locuteurs qui les produisent, ou plutôt qui les reproduisent. Dans la configuration intellectuelle de l'époque, où l'analyse du discours politique est une démarche de dévoilement idéologique, le fait de mettre au jour les régularités d'un discours est un moyen de montrer comment un sujet «est parlé» par une idéologie. Loin des considérations actuelles de la psychologie ou de la communication politique, pour lesquelles le discours résulte d'une intention ou révèle des stratégies, le discours est alors appréhendé comme l'expression d'un complexe idéologique qui transcende le sujet. La notion de «formation discursive», proposée par Michel Foucault, et retravaillée par Michel Pêcheux et d'autres figures emblématiques de l'École française d'analyse du discours, contribue à penser cet assujettissement idéologique à travers le langage.

D'une certaine manière, c'est un tel assujettissement que s'emploient à faciliter les locuteurs auxquels nous nous intéressons dans la seconde partie de ce texte («Les instruments de la stabilisation de l'expression : mettre en place un vocabulaire autorisé»). De façon certaine, c'est un tel assujettissement que dénoncent les locuteurs dont nous étudions les propos critiques dans un troisième temps («Des dispositifs pour déconstruire l'autorité en discours : subvertir le figement de l'expression »).

Pour notre part, en considérant que le figement a effectivement à voir avec des phénomènes puissants de domination et d'émancipation, nous prolongeons les réflexions menées par l'École française d'analyse du discours dans les années soixante / quatre-vingt. Parallèlement, nous déplaçons les terrains d'observation du côté de la réflexivité langagière et de sa mise en œuvre, en ce sens qu'il s'agit moins d'observer des récurrences d'expression que de voir comment de telles récurrences sont travaillées et explicitement commentées.

\section{Un domaine de recherche pour les sciences du langage, un ensemble de pratiques pour les acteurs sociaux}

Aujourd'hui, la notion de figement continue d'être mobilisée pour l'analyse des discours politiques et institutionnels, notamment à travers l'étude des slogans et des formules, ainsi que nous l'avons fait nous-même autour de cette dernière notion (Krieg-Planque, 2009). Mais il est clair que le figement concerne aussi plus globalement les sciences du langage dans leur ensemble et dans leur diversité. L'intérêt pour l'idéologie s'estompe alors, bien entendu, au profit d'un recentrage sur différentes autres questions que soulèvent la langue et le discours. De fait, le figement apparaît comme un phénomène sur lequel travaillent toutes les ramifications des sciences du langage : lexicologie, syntaxe, sémantique, terminologie, traductologie, didactique, rhétorique, pragmatique, 
stylistique... Dans le cadre de cet article, nous n'avons pas la place d'établir une bibliographie sur la notion de figement, mais nous pouvons renvoyer aux récents volumes coordonnés par Jean-Claude Anscombre et Salah Mejri (2011) d'une part, et par Laurent Perrin (2013) d'autre part.

Nous souhaitons en revanche faire part de quelques observations distanciées sur la notion de «figement » telle qu'elle est travaillée en sciences du langage. La conjonction de la grande finesse des descriptions linguistiques et du développement du traitement automatique des langues naturelles permet de mener des recherches approfondies sur les diverses manifestations du figement. Grâce à l'annotation des corpus, il est possible de formuler des requêtes qui permettent à la machine de repérer des types de récurrences très précises, y compris selon des critères sémantiques.

De telles possibilités sont un progrès pour la recherche en sciences du langage. Néanmoins, elles peuvent amener à faire oublier que si les phénomènes de figement sont des faits de langue, ils sont aussi des faits de langue en relation avec des pratiques sociales. Nous voulons dire par là que la possibilité de la langue de "faire figement» (sous les formes extrêmement variées des noms composés, des idiotismes, des proverbes...) est une possibilité dont se saisissent, de manière organisée ou non, des acteurs sociaux individuels ou collectifs. Si «transition énergétique » fonctionne comme syntagme figé dans l'univers discursif des années deux mille dix, ce n'est pas seulement en vertu de l'attirance mutuelle des nominalisations d'action et des adjectifs dénominaux, mais aussi grâce à la force rhétorique de cette expression dans un espace public historiquement donné. Si «ll faut manger pour vivre et non pas vivre pour manger» est constitué en proverbe, ce n'est pas uniquement du fait de ses qualités formelles en tant que chiasme, mais également grâce à sa répétition comme transmission sociale d'une morale des conduites.

Dans la suite de cet article, c'est en tant qu'il est pris dans une pratique sociale que le figement - puis sa subversion - va retenir notre attention. Il s'agit de montrer comment des acteurs sociaux mettent en place des instruments, des genres et des dispositifs dont l'un des effets est qu'ils produisent du figement. Les phénomènes de figement, alors, apparaissent comme le résultat conjoint d'une ressource de la langue et d'une organisation sociale.

Laissant à présent derrière nous le cadre théorique qui motive notre réflexion, nous nous employons, dans les pages qui viennent, à valoriser quelques exemples : nous montrons comment, très concrètement, des locuteurs créent ou renforcent le caractère figé de certaines séquences, ou au contraire tentent d'attirer l'attention sur la fausse évidence que présentent certaines suites verbales du fait de leur stabilisation.

En évoquant ces exemples, qui permettent d'identifier quelques points précis de l'intrication entre l'autorité en discours et le figement de l'expression, nous parcourons des domaines de savoirs et de pratiques professionnelles et 
sociales très diversifiés : terminologie, traductologie, lexicographie, normes d'autorégulation du secteur marchand, éducation populaire, usages militants d'Internet, littératie numérique...

\section{Les instruments de la stabilisation de l'expression : mettre en place un vocabulaire autorisé}

Dans les organisations, il existe divers types d'instruments qui, de fait, reviennent à stabiliser l'expression : «chartes de rédaction» dans certaines grandes entreprises, «manuels de style» des rédactions dans les médias, "guides de simplification linguistique» dans les administrations publiques, "codes de rédaction» interinstitutionnels de l’Union européenne, "glossaires» thématiques des organisations supranationales, «éléments de langage » gouvernementaux, « argumentaires» des partis politiques... Chaque secteur professionnel, chaque sphère d'activité et chaque appareil politique se conforme à des normes langagières et met en place des instruments qui explicitent au moins partiellement ces normes. Deux de ces types d'instruments sont présentés ci-après, à titre d'exemples.

\section{Produire une terminologie à même de circuler dans des espaces colingues : les bases de données terminologiques multilingues}

Un premier type de dispositif qui retient notre attention est celui des bases de données terminologiques multilingues. Celles-ci consistent en un rassemblement structuré d'informations concernant l'ensemble des «termes» identifiés comme tels par une institution donnée (un «terme» étant, en terminologie, un mot ou groupe de mots auquel il est assigné un contenu délimité en relation avec un domaine d'usage). L'unité élémentaire de l'information dans la base de données est une «fiche de terme» ou une «notice», laquelle comporte différentes rubriques (définition du terme, source de la définition, propositions de traduction dans des langues cibles, note de fiabilité de ces traductions, traductions déconseillées, date de mise à jour de la notice...). Ces notices sont créées et renseignées par les contributeurs de la base : terminologues, traducteurs, rédacteurs, juristes-linguistes et autres «travailleurs du langage» dans l'institution. Exprimé autrement, on pourrait dire qu'une base de données terminologique multilingue est le stock informatisé des expressions reconnues parl'institution à un moment donné, comportant également des informations sur les traductions, les domaines de spécialité et les contextes d'emploi de ces expressions. Comme on le comprend, une base de données terminologie multilingue présente un fondement profondément normatif : il s'agit de répertorier les expressions autorisées, ainsi que leurs définitions acceptables et leurs traductions recommandées. 
Différentes bases de données terminologiques multilingues existent de par le monde : Termium Plus et Termicom pour le Canada, IATE pour l'Union européenne... C'est cette dernière que nous évoquerons, non seulement par intérêt personnel pour l'espace européen, mais aussi parce qu'IATE est accessible librement sur Internet : nous pouvons ainsi inciter le lecteur à aller expérimenter par lui-même un riche dispositif que nous ne faisons qu'effleurer'1.

IATE (Inter-Active Terminology for Europe) a commencé à fonctionner en 2004, par fusion des bases de données préexistantes de différentes institutions de l'Union (Eurodicautom pour la Commission, Euterpe pour le Parlement, TIS pour le Conseil...). Proposant des traductions dans les langues officielles de l'Union, soit actuellement 24 langues, la base IATE contient environ 8,5 millions de termes. Elle permet à l'ensemble des terminologues et traducteurs des institutions européennes de mutualiser leur travail sur les notices. Mise à disposition de tous les personnels de l'Union, mais aussi du grand public depuis 2007, elle apparaît comme un instrument essentiel du contrôle de la créativité verbale tel qu'évoqué au début de cet article.

Parmi les millions de termes qui font l'objet d'une notice dans IATE, beaucoup sont des lexèmes construits ne comportant qu'un seul mot graphique («densitomètre », « enneigement», «éducation »...), et, par ailleurs, beaucoup d'unités lexicales complexes correspondent clairement à des langues de spécialité (« contrat conditionnel », " sylviculture à courte rotation », «fusion thermonucléaire contrôlée »...). Mais la base comporte aussi des termes relevant avant tout du vocabulaire socio-politique, et renvoyant parfois à des concepts contestés ou discutés sur d'autres scènes : «démocratie participative », «développement durable», "diversité culturelle »... Ainsi, jusqu’à un certain point, IATE participe à une naturalisation de certaines expressions et de certains concepts $^{2}$. Dans tous les cas, par son principe de fonctionnement, le dispositif consiste, de fait, à faire exister certaines collocations, et à en méconnaître d'autres. Ainsi, les termes «économie sociale de marché », «patrimoine culturel immatériel » et «résilience de l'écosystème» existent dans la base, où ils font l'objet d'une notice. À l'inverse, les formulations «altermondialisme», «collectif de travail » ou « domination masculine » sont totalement absentes de IATE, ce que l'on peut raisonnablement interpréter comme relevant d'une trace des orientations idéologiques des élus et fonctionnaires européens.

Bien entendu, IATE doit être considérée avant tout comme un outil professionnel des services linguistiques de l'Union, dans le cadre de la division du travail qu'impose la dimension colingue des textes et discours produits par les institutions internationales ou supranationales. Il faut en outre rappeler que les contri-

1. L'utilisateur effectue ses requêtes à partir de l'interface consultable à l'adresse http://iate. europa.eu. Les données que nous mentionnons dans cet article résultent d'une consultation de IATE au 13 février 2014 .

2. Sur les enjeux politiques de la terminologie multilingue, on pourra voir Raus, 2013. 
buteurs d'IATE se contentent d'enregistrer les usages attestés qu'ils observent dans des textes et discours. Néanmoins, on ne peut manquer de remarquer qu'une telle base de données consiste, de fait, à réduire la diversité des formulations disponibles et celle de leurs traductions. L'institution, de ce fait, apparaît comme créatrice d'unités phraséologiques, qu'elle rend disponibles pour la reprise et la circulation. Enfin, il faut souligner que l'enrichissement d'IATE est une tâche routinière pour les «travailleurs du langage » de l'Union européenne : de nouveaux termes sont ajoutés quotidiennement, et le contenu des notices est constamment actualisé. La mise en place d'expressions figées apparaît ainsi comme une activité ordinaire du travail des institutions.

\section{Cadrer l'usage institutionnellement utile des expressions : les recommandations en matière de vocabulaire}

Le deuxième type d'instrument qui nous intéresse au titre d'illustration regroupe des documents d'autorégulation à travers lesquels sont données des préconisations en matière de vocabulaire. Par "autorégulation », il faut entendre l'ensemble des règles qu'une sphère d'activité se donne à ellemême, au-delà de ce que les contraintes légales lui imposent (il peut s'agir par exemple de proscrire les réunions de travail après 18 heures, de manière à préserver la vie personnelle des salariés). Ce que l'on appelle ailleurs la «responsabilité sociale des entreprises » relève en partie de ce type de comportement volontaire des organisations.

Pour les acteurs qui participent à ces démarches d'autorégulation, il s'agit de mettre en place des «bonnes pratiques », c'est-à-dire des règles non imposées par les contraintes légales, et qui donnent une image positive de ces acteurs (y compris à travers des «labels» ou des «chartes», par exemple). Ces «bonnes pratiques» sont parfois l'antichambre de la fabrique de nouvelles règles juridiquement contraignantes : certains chercheurs parlent alors de «droit mou » ou de «droit souple» («soft law»). Là encore, nous ne pouvons développer le sujet3.

La plupart des recommandations relevant de «bonnes pratiques» à suivre ne portent pas, ou du moins pas directement, sur des questions langagières, mais plutôt sur l'éthique des affaires, les relations avec les syndicats, les rapports avec la sous-traitance, ou encore la place faite aux personnes discriminées. Ce sont bien entendu uniquement les questions directement liées à l'usage de la langue qui nous occupent ici.

Dans le domaine de la dénomination des personnes, il est courant d'ironiser sur l'apparence technocratique ou la dimension euphémisante de formulations telles que «personne souffrant de schizophrénie» (à la place de «schi-

3. Le lecteur pourra se référer par exemple à Cochoy, 2007. 
zophrène ») ou "personne en situation de handicap» (pour «handicapé»). En réalité, ces dénominations correspondent à des logiques d'action cohérentes: la maladie est présentée comme quelque chose qui vient de l'extérieur de la personne, laquelle peut donc ainsi peut-être voir son état s'améliorer; ou encore, la personne est présentée comme ne se résumant pas à son handicap, et ne partageant que certains aspects de son existence avec des personnes en situation analogue. Concernant le sujet de cet article, ces changements de dénominations reviennent, de fait, à faire un usage plus systématique de collocations.

Dans le secteur de la publicité, l'ARPP (Autorité de régulation professionnelle de la publicité) mène une surveillance étroite des expressions utilisées dans les textes publicitaires, de manière à anticiper les critiques. Dans le domaine de l'environnement et de l'énergie, l'ADEME (Agence de l'environnement et de la maîtrise de l'énergie) édite régulièrement des recommandations langagières. Se mettre d'accord sur des «bonnes pratiques » en matière de discours, c'est se mettre d'accord sur des conventions d'expressions.

L'exemple qui vient est particulièrement significatif de la façon dont une «surveillance du discours» effectuée dans un cadre d'autorégulation peut conduire à produire des expressions figées. Dans le document qui suit, les valeurs de conseil et de suggestion caractéristiques de l'autorégulation, et qui sont plutôt celles que l'on relève dans les documents de l'ARPP ou de l'ADEME, s'effacent derrière une valeur injonctive : c'est alors une injonction au figement que l'on observe, clairement exprimée ici par le fait qu'un terme (ici « durable») ne doit être employé qu'en cooccurrence avec un autre (ici « développement»).

En 2012, le ministère de l'Écologie, du Développement durable, des Transports et du Logement et le ministère de l'Économie, des Finances et de l'Industrie coéditent une brochure intitulée Guide pratique des allégations environnementales à l'usage des professionnels et des consommateurs 4 . Cette brochure de 42 pages expose, sous forme d'un lexique de 15 entrées («Durable», «Ecoconçu », « Naturel », « Recyclable »...), les façons les plus recommandables de recourir à ces vocables. L'entrée « Durable» de ce guide débute ainsi : « Le terme "durable" ne doit être employé qu'en référence au "développement durable", sauf s'il qualifie un produit qui dure plus longtemps.»

Plus loin sur la double page, après un rappel de la définition canonique du "développement durable» telle que fournie par le rapport Brundtland de 1987, puis une présentation des «trois piliers du développement durable », le Guide réitère : "Seule l'expression "développement durable" doit être employée. "Durable", seul, peut néanmoins qualifier un produit qui dure plus longtemps: par exemple, une pile, une lampe...»

Nous ne saurions pas mieux montrer qu'il existe des injonctions au figement,

4. En ligne: http://www.economie.gouv.fr/files/guide_allegations_31janv.pdf(consulté le 13 février 2014). 
au sens où certains acteurs émettent des consignes de limitation de la créativité verbale (exercée notamment sous les formes diversifiées de la néologie, du défigement...).

Ainsi, pour des raisons qui ne relèvent plus cette fois de la division du travail en matière de rédaction-traduction des discours institutionnels (exemple d'IATE), mais pour des motifs de renforcement de leur acceptabilité sociale, des acteurs peuvent être amenés à encourager la production, ou plutôt la reproduction, de formulations stabilisées.

\section{Des dispositifs pour déconstruire l'autorité du discours : subvertir le figement de l'expression}

Nous nous intéressons à présent à des démarches qui, comme en écho aux précédentes, s'emparent de certains discours aux fins d'en saper l'autorité. Engagés dans une perspective critique, les locuteurs étudiés mettent explicitement en scène le fait que ces discours sont en quelque sorte «cousus » à partir d'expressions stéréotypées : en exhibant les discours adverses en tant que suites de mots prévisibles, les locuteurs entreprennent d'en déconstruire les effets d'évidence. Ce faisant, ils se donnent en général à voir comme participant à une action de conscientisation.

La dimension subversive du défigement (dans les titres de presse, dans la parodie littéraire...) a déjà été amplement étudiée, notamment depuis un article de Pierre Fiala et Benoît Habert (1989) paru dans cette même revue Mots. Les langages du politique (revue Mots, à l'époque), et qui s'intitulait précisément "La langue de bois en éclat». Pour notre part, nous mettrons l'accent non pas sur des séries d'énoncés relevant du défigement (défigements de slogans, de déclarations célèbres...), mais sur les principes de fonctionnement de certains genres et dispositifs de subversion, dont deux sont évoqués à présent, là aussi au simple titre d'illustration et faute de place pour diversifier les exemples.

\section{Réagencer des unités ou des collocations : les générateurs d'énoncés}

Depuis la généralisation d'Internet, et particulièrement depuis celle du Web dynamique et participatif ( Web 2.0») dans le milieu des années 2000, les utilisateurs qui sont dotés d'une certaine littératie numérique ont la possibilité de recourir, en les combinant, aux capacités algorithmiques de l'informatique, d'une part, et aux possibilités d'autopublication offertes par Internet, d'autre part. En s'appuyant sur cette double possibilité, certains utilisateurs ont ainsi mis en ligne des dispositifs que nous appelons ici des « générateurs d'énoncés».

Ceux-ci consistent à agencer de manière aléatoire des unités appartenant à 
un certain nombre de paradigmes syntaxico-sémantiques prédéterminés. Par exemple, on agencera n'importe quel syntagme prépositionnel exprimant la finalité («pour », « afin de », « de manière à »...) avec n'importe quel verbe à l'infinitif exprimant une amélioration («optimiser», «embellir», «bonifier»...) et avec n'importe quel syntagme nominal référant à une procédure («les process industriels », «l'ordre d'exécution des programmes», «la chaîne de commandement »...). On formera ainsi des énoncés tels que : «De manière à optimiser les process industriels », » De manière à bonifier la chaîne de commandement, «Pour optimiser l'ordre d'exécution des programmes»...

Dans leur architecture, les générateurs d'énoncés s'appuient sur la ressource de la langue comme système, laquelle autorise la commutation à l'intérieur d'un même paradigme : ce principe de commutation est l'un de ceux, avec quelques autres (récursivité...), qui permettent à une communauté parlante de créer un nombre infini d'énoncés à partir d'un stock limité de mots et de règles. Les générateurs d'énoncés exploitent donc une possibilité qui a déjà été bien repérée par les poètes et joueurs de la langue, et qu'illustrent remarquablement diverses œuvres des membres de l'Oulipo, tel que Cent mille milliards de poèmes de Raymond Queneau.

Mais, ici, ces possibilités sont à la fois automatisées et mises au service d'une critique politique ou idéologique : même s’ils peuvent apparaître fondamentalement comme poétiques, ces générateurs d'énoncés sont avant tout tournés vers la dérision critique. Comme l'exemple imaginé ci-dessus permet de le pressentir, l'exploitation des ressources de la langue peut facilement se prêter, à travers des dispositifs spécifiques, à une mise en avant dénonciatrice des unités phraséologiques, des routines d'écritures et des stéréotypes d'expression qui forment certains discours. De fait, les générateurs d'énoncés s'attaquent au caractère préfabriqué de certains discours publics, dont ils rendent observable, de manière à la fois spectaculaire et concrète, la dimension prévisible.

Sur un mode non automatisé, Franck Lepage s'est rendu célèbre à travers une brève séquence de sa «conférence gesticulée» de 2007 portant sur l'éducation populaire5. Cet extrait de cinq minutes, déposé sur une plateforme de partage vidéo en 2009 sous le titre "Langue de bois », et dont les consultations par des internautes se montaient à environ 450 ooo vues début $2014^{6}$, donne à voir la façon dont la combinaison d'unités lexicales en nombre limité (« diagnostic partagé», «lien social», «participation», «proximité»...) permet de produire des propos phraséologiques convenus.

5. Franck Lepage, «Inculture(s). Petits contes politiques et autres récits non autorisés », «1. “L'éducation populaire, Monsieur, ils n'en ont pas voulu..." Une autre histoire de la culture », enregistré à l’Espace Jemmapes, Paris, 21 mai 2007, DVD en diffusion non commerciale, par la SCOP «Le Pavé», durée 2 h 17. Voir Krieg-Planque, 2012.

6. Franck Lepage, «Langue de bois »: http://www.youtube.com/watch?v=oNJo-E4MEk8 (consulté le 13 février 2014). 
Mais une multitude d'activistes ont automatisé la ressource, permettant à l'utilisateur d'être également un expérimentateur actif de la production de discours stéréotypés, dont cet utilisateur peut ainsi mieux comprendre les ressorts. Par exemple, alors que Frédéric Lefebvre était porte-parole de l'UMP, un site Web intitulé Le Lefebvrotron7 proposait sur un mode ironique un " générateur de communiqués », grâce auquel était mis en scène le caractère prévisible du discours du porte-parole de l'UMP, et en conséquence une certaine vacuité du discours de la communication politique. Ou bien, sur un mode encore plus subversif, l'agence de communication alternative Formes Vives ${ }^{8}$ propose un site Web à partir duquel l'utilisateur peut générer automatiquement non seulement des logos de collectivités locales, mais surtout les discours de présentation de ces logos, qui parodient les discours des professionnels de la communication : c'est le caractère interchangeable, et par là profondément inconsistant, des discours des agences de communication qui est visé. D’autres dispositifs encore pourraient être mentionnés, tels que le Pipotron' ou le Générateur de langue de bois ${ }^{10}$ pour candidats aux élections présidentielles. Nous invitons le lecteur à aller expérimenter ces dispositifs.

À chaque fois, pour ces générateurs d'énoncés, il s'agit de délinéariser des segments préformés pour les réagencer autrement, de manière à constater ensuite que ce réagencement, tout en étant inédit, produit néanmoins des discours convenus : les segments apparaissent alors dans leur dimension stéréotypée, confirmant le sentiment rhétorique spontané qui confère à ces discours à la fois un caractère d'évidence et une absence de contenus susceptibles d'être contredits.

\section{Mettre en scène les passages obligés du discours : les cartes et plans du vocabulaire imposé}

Le deuxième genre que nous évoquons s'appuie sur les visualisations du langage (sous formes de cartes, de plans...) comme représentations symboliques. Les enjeux politiques des cartes et des plans ont déjà été largement étudiés par les historiens, les géographes, les politistes et les anthropologues. Nous nous bornons à signaler un type très spécifique d'usage, exclusivement lié à la question du discours. Pour le locuteur en posture réflexive engagé dans une démarche critique, le principe consiste ici à transposer sur une carte ou sur un plan la représentation qu'il se fait, ou du moins qu'il souhaite donner à voir, du discours qu'il dénonce.

7. Le Lefebvrotron, générateur de communiqués : http://lefebvrotron.fr (idem).

8. Formes Vives : http://formes-vives.org. Logo de ville : http://logo-de-ville.fr (idem).

9. Pipotron : http://www.pipotron.free.fr (idem).

10. Le générateur de langue de bois : http://www.presidentielle-2007.net/generateur-de-languede-bois.php (idem). 
Le plan publié sur le site Web ArTerrOriste ${ }^{11}$ offre un exemple remarquable de ce que peut être une représentation graphique du sentiment rhétorique dans le cadre d'une critique des discours politiques : s'y combinent de façon particulièrement expressive la performance artistique et la critique idéologique. Comme pour tous les exemples précédents, nous invitons le lecteur à aller explorer le site Web et nous limitons à quelques commentaires analytiques essentiels.

Dans une rubrique intitulée "Les mots du pouvoir. La novlangue néolibérale », le rédacteur du site ArTerrOriste propose de télécharger un plan, que les codes graphiques assimilent aisément à un plan de métro parisien, sur lequel sont reliées autant de formulations supposées caractériser le discours néolibéral. "Gouvernance », «Modèle danois», «Démocratie actionnariale », «Choc des civilisations», «Monde de plus en plus complexe», et quelques dizaines d'autres expressions (301 unités au total) sont données à voir comme autant de passages obligés des discours pour les locuteurs qui défendent les intérêts du néolibéralisme : la production discursive de ces derniers se limiterait à relier ces points les uns aux autres, autrement dit à coudre des pièces préfabriquées selon une combinatoire relativement fermée. À l'instar du parcours contraint, routinier et aliénant qu'il effectue en tant qu'usager des transports dans les couloirs du métro parisien, le lecteur du plan conçu par ArTerrOriste "pourra à loisir recomposer des discours», comme il est indiqué, mais cette fois-ci dans une démarche de conscientisation.

Pour notre propos, le fait que les étiquettes associées à chacun des 301 points de passage (qui sont représentées comme autant de noms de stations de métro) soient parfois des unités lexicales complexes, voire des slogans («Il n'y a pas d'alternative »), est bien entendu intéressant : ce fait signale que le discours à combattre est saisi «par ses figements».

Mais tout aussi notable est le fait que ces unités sont présentées à travers les liens qu'elles entretiennent les unes avec les autres, lesquels apparaissent comme des relations surdéterminées par les lignes et les correspondances permises par la structuration du réseau. Par exemple, la ligne n ${ }^{0} 13$ (qui a pour nom «Le management de l'individu ») permet d'utiliser sans passer par une correspondance les unités «Employabilité», «Savoir être » ou encore « Formation tout au long de la vie», qui sont disposées le long de cette ligne. En revanche, pour atteindre les unités «Attractivité», «Compétitivité» ou «Innovation », situées sur la ligne n 2 (appelée «L'esprit de l'entreprise»), il sera nécessaire de prendre la correspondance à l'unité «Capital humain», qui est commune aux lignes $n^{\circ} 2$ et $n^{\circ} 13$. Ainsi, ArTerrOriste expose par les moyens de la visualisation cartographique ce qui, dans un discours, relève du conditionnement et de la prévisibilité : la proximité relative des mots les uns avec

11. ArTerrOriste $: \mathrm{http}: / /$ arterroriste.pagesperso-orange.fr [consulté le 13 février 2014]. 
les autres dans le réseau urbain souterrain, qui trahit leur interdépendance et l'impossibilité à sortir de ce circuit du vocabulaire imposé, révèle des intuitions qu'objectivaient les travaux de lexicométrie inaugurés dans les années soixante / soixante-dix sur les phénomènes de cooccurrence.

Enfin, on ne saurait manquer de remarquer que le plan de la «novlangue néolibérale » imaginé par ArTerrOriste figure en surimpression de trois lettres en majuscules, qui transparaissent dans un gris clair à peine visible, comme en filigrane, sur l'essentiel de la surface du plan. Ces trois lettres sont «LQR ». La phraséologie de la novlangue néolibérale s'inscrirait donc, d'après l'auteur, sur fond de $L Q R$. Or ce sigle est celui par lequel l'éditeur et essayiste Éric Hazan appelle ce qu'il estime être la Lingua Quintae Respublicae (la langue de la Cinquième République), et qu'il rapproche très explicitement de la LTI, la langue du Troisième Reich décrite par Victor Klemperer.

Pour notre part, il n'est évidemment pas question de prendre en charge une telle analogie entre les discours totalitaires des années trente-quarante et les discours politiques et institutionnels qui nous sont contemporains. En revanche, en tant qu'analyste, il nous semble remarquable que, par-delà la singularité des situations historiques considérées et la pluralité des langues concernées, la construction et la déconstruction de l'autorité en discours invitent toutes deux à mettre la question du figement au cœur de la réflexion. Dans leur travail de réflexivité langagière, les locuteurs qui donnent du crédit à l'institution œuvrent à produire de la régularité, à limiter le surgissement de l'inattendu, à contrôler l'étendue de la créativité verbale. En écho, les locuteurs qui exercent leur sentiment linguistique dans une perspective critique associent spontanément l'imposition de l'autorité à la réduction des possibles dans l'ordre du discours, et expriment leur remise en cause de l'autorité à travers une subversion de l'expression comme suite de formulations prévisibles.

\section{Références}

Anscombre Jean-Claude, MejRI Salah éd., 2011, Le figement linguistique. La parole entravée, Paris, Champion.

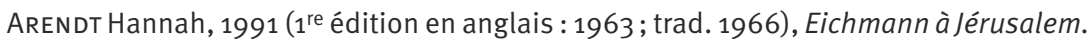
Rapport sur la banalité du mal, Paris, Le Seuil.

Cochor Franck, 2007, «La Responsabilité Sociale de l’Entreprise comme “représentation" de l'économie et du droit», Droit et Société. Revue internationale de théorie du droit et de sociologie juridique, n065, p. 91-101.

Fiala Pierre, HABERT Benoît, 1989, "La langue de bois en éclat. Les défigements dans les titres de presse quotidienne française», Mots, n ${ }^{0}$, p. 83-99.

FiALA Pierre, 1987, «Pour une approche discursive de la phraséologie. Remarques en vrac sur la locutionnalité et quelques points de vue qui s'y rapportent, sans doute », Langage et Société, nº 42, p. 27-44. 


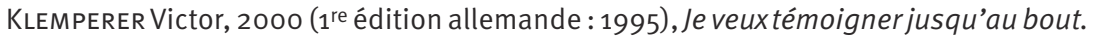
Journal 1941-1945, Paris, Le Seuil.

KRIEg-Planque Alice, 2009, La notion de «formule» en analyse du discours. Cadre théorique et méthodologique, Besançon, Presses universitaires de Franche-Comté.

— 2012, "La “conférence gesticulée” comme théâtre politique et expérience personnelle. Militantisme et travail de l'intime», Itinéraires. Littérature, textes, cultures, vol. II, p. 167-170.

Perrin Laurent éd., 2013, «Le figement en débat», Pratiques. Linguistique, littérature, didactique, nº159-160.

Raus Rachele, 2013, La terminologie multilingue. La traduction des termes de l'égalité H/F dans le discours international, Bruxelles, De Boeck.

SALEm André, 1987, Pratique des segments répétés. Essai de statistique textuelle, Paris, INaLF-Klincksieck.

TouRnIER Maurice, 1985, "Texte "propagandiste" et cooccurrences. Hypothèses et méthodes pour l'étude de la sloganisation », Mots. Ordinateurs, textes, sociétés, $n^{0} 11$, p. $155-187$. 